\title{
INVESTIGACION
}

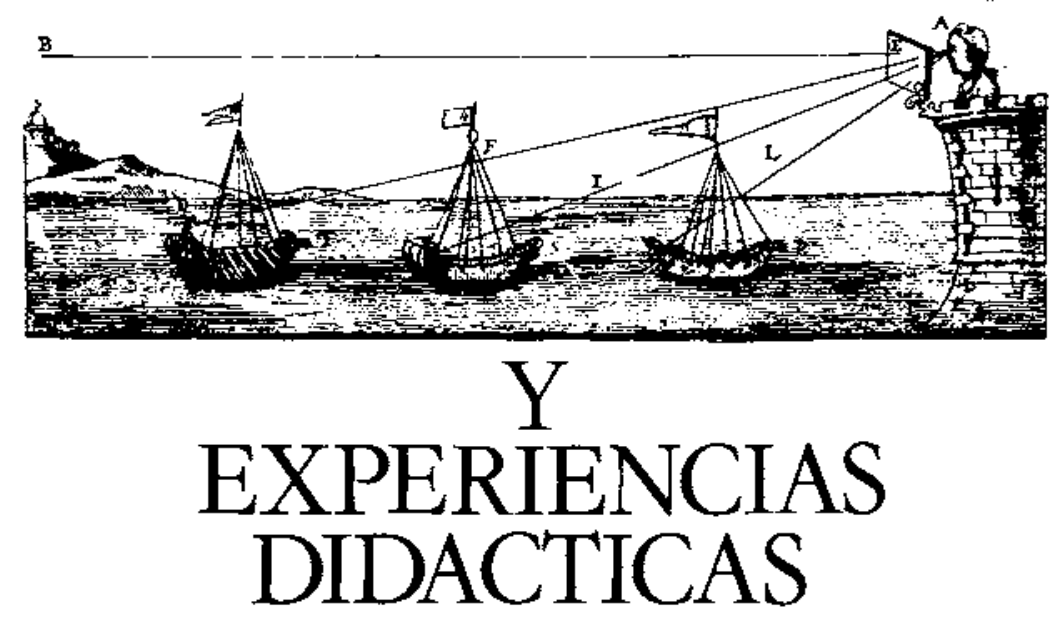

\section{EL CONOCIMIENTO ACERCA DE LAS MATEMÁTICAS Y LAS PRÁCTICAS DE ENSENANZA}

\author{
SÁNCHEZ GARCÍA, M̃ V. y LLINARES CÍSCAR, S. \\ Departamento de Didáctica de las Ciencias (Matemáticas). Universidad de Sevilla.
}

\section{SUMMARY}

The individual and contextual influences that two periods of school experience have over the way in which two prospective teachers build their personal view of Mathematics and its teaching are studied. In particular, the important role played by the perceived knowledge of the subject matter knowledge is shown. Also, the strategies used in trying to give coherence and meaning to the experimented changes are discussed.

\section{INTRODUCCIÓN}

Durante los últimos años hemos desempeñado nuestro trabajo como profesores de Didáctica de las Matemáticas en la E.U. de Magisterio de la Universidad de Sevilla. En nuestra Escuela, las prácticas de enseñanza se desarrollan en los cursos Segundo y Tercero, y los profesores de Ios citados cursos, además de impartir sus asignaturas correspondientes, están encargados de la supervisión y evaluación de las prácticas de enseñanza del grupo de alumnos que se les asigna.

EI hecho de ser profesores de una asignatura de Didáctica y tutores de prácticas nos ha puesto de manifiesto, de una manera más intensa, si cabe, la necesidad de vincular la teoría y la práctica en la formación de los 
futuros maestros. Además, la constatación de la complejidad de las situaciones de enseñanza y de los conocimientos desarrollados por los profesores en estas situaciones nos ha llevado a interesarnos por el papel que juegan las prácticas de enserianza en la transformación de los conocimientos teóricos en prácticos, de nuestros alumnos, futuros maestros (Llinares y Sánchez 1986).

La investigación que a continuación desarroltamos no ha nacido por tanto de la teoría, sino que tiene sus orígenes en nuestra tarea diaria.

\section{MARCO TEÓRICO}

El contexto teórico en el que desarrollamos este trabajo se encuadra dentro del paradigma del pensamiento del profesor (Clark y Peterson 1986, Shulman 1986a, Marcelo 1987), y dentro de este paradigma, nos centramos en cuáles son "las formas en que los profesores adquieren activamente y utilizan el conocimiento, y las circunstancias que afectan su adquisición y empleo" (Calderhead 1987, p. 5).

Uno de los principales resultados de las investigaciones sobre el pensamiento de los profesores ha sido precisamente el profundizar sobre este conocimiento, y sobre el importante papel que juega en los procesos de enseñanza. Shulman (1987a) afirma que el motivo para resaltar el papel fundamental del conocimiento, más que el comportamiento de los profesores, es precisamente el carácter esencial de la adaptabilidad de los maestros al contexto.

En relación al contenido de este conocimiento, el mismo autor (Shulman 1987b, Wilson et al. 1987) ha señalado distintas categorías, entre las que se encuentra el conocimiento de la materia específica, en nuestro caso las Matemáticas.

Centrándonos en el conocimiento de contenido de las Matemáticas, podemos establecer una subcategorización (Shulman 1986b) en la que se podría considerar el conocimiento de la materia específica "per se", el conocimiento de contenido pedagógico específico de la misma y un conocimiento curricular, que abarca no sólo el específico de la materia, sino también el de otras materias en el mismo curso y en cursos anteriores y posteriores.

Muy recientemente, Ball (1989) ha distinguido tres componentes del conocimiento de la materia específica (Matemáticas). En primer lugar, identifica aquellos aspectos asociados con lo que convencionalmente se entiende por conocimiento de las Matemáticas (conocimiento sustantivo).

En un segundo grupo incluye lo que significa saber y hacer Matemáticas, qué es una demostración, quiên tiene el poder de validación, de dónde provienen, etc. (conocimiento acerca de las Matemáticas).
Finalmente, la tercera componente se refiere a la disposición hacia las Matemáticas, que englobaría los sentimientos emocionales hacia las mismas. Para esta autora, el conocimiento de las Matemáticas es una interrelación de los tres aspectos citados. La descripción de las diferentes categorías del conocimiento profesional del profesor de Matemáticas (Llinares y Sánchez 1990) aporta datos de interés para el diseño de programas de formación de profesores.

Dada la complejidad del conocimiento, y retomando nuestro planteamiento inicial, la cuestion ante la que nos encontramos es cuáles serán los factores importantes en el desarrollo del conocimiento de los futuros maestros durante su período de formación.

Siguiendo una propuesta de Elbaz (Elbaz et al. 1986) podemos considerar entre ellos:

a) La clase de conocimientos que adquieren. Por ejemplo, respecto del conocimiento de contenido de las Matemáticas tienen que ser capaces de realizar una transformación del mismo y de su modo de utilizarlo, pasando de la teoría a la práctica, lo que supone un cambio en el modo de utilizar el conocimiento.

b) Los Iugares donde tiene lugar la instrucción y la práctica, ya que adquieren conocimiento en una institución (Universidad) para utilizarlo en otra (escuela).

c) Los papeles (roles) asumidos en el proceso de formación. En particular, puede verse de un modo muy distinto las Matemáticas como alumno y como maestro.

Por otro lado, según el punto de vista que se adopte, pueden distinguirse distintas aproximaciones en el estudio de los complejos procesos que intervienen en la formación y desarrollo de los profesores (Veenman 1984, Feiman-Nemser y Floden 1986). Nosotros nos hemos centrado en los cambios de la persona social en su interrelación con el medio, interesándonos por lo tanto en los procesos de socialización de los futuros profesores, entendida la socialización en un sentido amplio (Zeichner y Gore 1989), y en las estrategias que adoptan en el contexto de situaciones específicas (Lacey 1977).

Este último autor introdujo el concepto de estrategia social para referirse a un sisterna de ideas y acciones que un individuo desarrolla intencionadamente en determinadas situaciones. El tipo de estrategia que desarrolla cada individuo depende de cómo interprete su situación y de su capacidad para llevarla a cabo. Lacey distinguió tres tipos de estrategias:

- ajuste interno, en que el individuo acepta como propios los valores y metas de la institución, así como las restricciones que le son impuestas, estando además convencido de que son buenas.

-sumisión estratégica, en que se acepta la definición de la situación que hace la autoridad, y también las condiciones, pero se mantienen reservas privadas hacia ellas.

-redefinición estratégica, que corresponde al caso en 
que el individuo trata de producir cambios, aun sin tener poder formal para ello.

Otra propuesta distinta en este mismo contexto ha sido realizada por Goodman (1985), quien enunció cuatro tipos de reacciones de los profesores y los profesores en formación a las normas institucionales: aceptación pasiva, aceptación activa, resistencia latente y resistencia abierta. Las denominaciones son suficientemente claras, y creemos que no necesitan aclaración.

Es en esta intersección de conocimiento y socialización en la que hemos situado nuestro trabajo, que a continuación vamos a desarroliar.

\section{PROPÓSITOS E HIPÓTESIS}

El propósito general de nuestra investigación ha sido estudiar las concepciones que acerca de las Matemáticas y su enseñanza tienen los maestros en formación, así como la influencia que sobre los mismos ejercen los periodos de prácticas docentes. La importancia de estas concepciones ha sido señalada por Cooney (1980) y Thompson (1984). Esta última afirma que "existen fuertes razones para creer que en las Matemáticas las concepciones del profesor y su enseñanza juegan un papel importante, afectando su importancia como mediador primario entre la materia y los que aprenden" (Thompson 1984, p. 105).

Si consideramos que las concepciones de los futuros maestros acerca de las Matemáticas y su enseñanza influencian su labor como profesores, está claro que la manera en que estas concepciones se desarrollan, en qué međida son vulnerables al cambio, y la interacción entre ellas y las prácticas docentes (Llinares 1989, Sánchez y Lilinares 1988) tienen implicaciones para los programas de formación de profesores (Harvey 1986).

En este punto, es importante señalar el hecho de que las experiencias y estudios realizados en otros países acerca de los períodos de prácticas son difícilmente trasladables, dadas las peculiaridades, incluso locales, de nuestro sistema de formación de profesores. Como Zeichner y Gore (1989) han señalado, se debe ser muy prudente en trasladar las investigaciones de un país a otro de forma lineal.

Como hipótesis básica de nuestro trabajo, adoptamos el punto de vista de que la forma de entender la realidad y las acciones presentes o futuras de los maestros en formación están guiadas por una interpretación personal de su entorno. Además, admitimos que esta interpretación está estructurađa por el conjunto de constructos de los individuos, de acuerdo con la Teoría de los Constructos Personales (T.C.P.) de Kelly (1955).

Esta teoría, basada en la posición filosófica del alternativismo constructivista, considera que todas nuestras interpretaciones actuales del entorno que nos rodea están sujetas a cambio. Cada persona posee un modelo de la realidad que utiliza para predecir hipótesis, que son después verificadas. Este proceso puede llevar, en su caso, a una alteración del modelo para adecuarlo a los resultados.

La T.C.P. proporciona un modo sistemático de đescubrir la realidad, lo que se hace en términos de imágenes dicotómicas, denominadas constructos. Los constructos de una persona constituyen una red característica de la misma, que está en continua evolución. Esta red es Ia que determina en qué forma percibe la persona la realidad, y anticipa hechos futuros.

En concordancia con Io anteriormente expuesto, hemos admitido en nuestro trabajo que los futuros maestros no son meros recipientes pasivos de conocimiento matemático, y que tienen la posibilidad, al menos en cierto grado, de acceso a su pensamiento profesional y que éste puede verbalizarse.

\section{METODOLOGÍA}

Los participantes fueron dos alumnos de la E.U. de Magisterio de la Universidad de Sevilla, de la especialidad de Ciencias, pertenecientes a un grupo de ocho que colaboraron en nuestra investigación.

El instrumento fundamental utilizado en nuestra investigación fue la técnica de rejillas, asumiendo explícitamente los principios básicos de la T.C.P. en que se basa. Existen otras importantes razones metodológicas para escoger dicha técnica (van Hunen 1986). Por un lado, el método permite obtener información acerca de los esquemas cognitivos de otras personas, desde los propios sistemas de referencia de éstas. Por otro, permite gran variedad de técnicas de análisis para obtener información.

Además de las rejillas se emplearon otros dos instrumentos en la recogida de datos: el diario y la entrevista. Los motivos para utilizar estas tres técnicas combinadas son múltiples. En primer Iugar, tanto la entrevista como los diarios sirvieron de instrumentos para estimular con más fuerza la reflexión de los futuros maestros acerca de su experiencia. Permitían además obtener información de si eran conscientes de los cambios que experimentaban, de los conflictos que se les presentaban, de su forma de solucionarlos y de las explicaciones que daban de todo ello.

En segundo lugar, sirvieron para precisar los significados que los participantes adherían a las etiquetas verbales de los constructos, y delimitar si las interpretaciones que realizábamos eran correctas.

Por último, la distinta naturaleza de los tres instrumentos utilizados nos proporcionaba un método de triangulación metodologica (Cohen y Manion 1980) que nos permitía comprobar y aumentar la validez de los resultados (Morine-Dershimer 1983).

El proceso de recogida de datos se desarrolló a lo largo de dos años (figura 1). En una primera entrevista, 


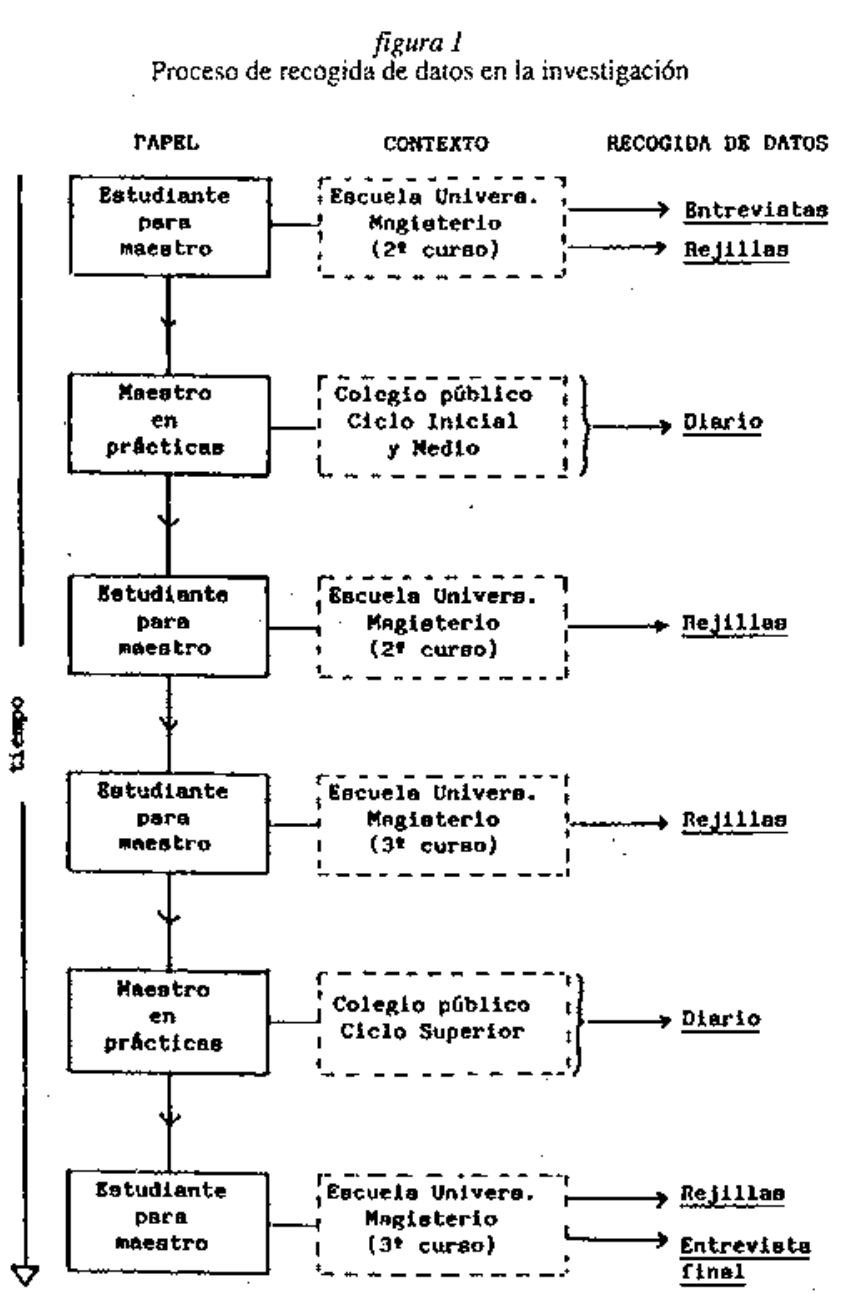

además de invitarles a participar y explicarles los puntos fundamentales alrededor de los que giraba la investigación, se les animó a reflexionar sobre la misma (Erickson 1986). A lo largo de las semanas siguientes se llevaron a cabo una serie de entrevistas, en las que se discutieron los elementos representativos que deberían figurar en cada una de las rejillas, referentes a los distintos temas objeto del estudio.

En relación a los constructos, se decidió utilizar una técnica mixta, incorporando algunos, procedentes de la bibliografía sobre el tema (McQualter 1986), que fueron asumidos por el grupo, y otros elicitados por los propios alumnos. Se presentaban tríadas de elementos y se preguntaba al grupo en qué se parecían dos de ellos, que les hiciesen diferentes del tercero. Por ejemplo, en el caso del profesor de Matemáticas, se les presentaba, entre otras, la tríada formada por el mejor profesor de Matemáticas que hubiesen tenido, el mejor profesor de otra asignatura y el peor profesor de Matemáticas. Los participantes describieron a los dos primeros como motivadores, asignándole el polo contrario al tercero.

A partir de los elementos y constructos se elaboraron cinco rejillas, referentes a la actuación/gestión de la clase, a la enseñanza de la materia específica Matemá- ticas, el profesor en la enseñanza de las Matemáticas, las Matemáticas como asignatura y como parte del currículo. Las rejillas tomaron forma de matriz, situándose los elementos en las columnas y los constructos en las fílas. Cada una de estas rejillas en blanco fue completada por cada uno de los participantes en cuatro ocasiones, tal y como indicamos en la figura 1, siguiendo un procedimiento elegido por ellos de situar una $\mathrm{V}$ cuando el elemento era valorado con el polo del constructo de la izquierda, una $X$ si lo era el de la derecha y VX si la valoración era intermedia.

\section{ANÁLISIS DE LOS DATOS}

Una vez completadas las rejillas se procedió a su análisis (Fransella y Bannister 1977, Pope y Keen 1981). El análisis directo de los datos permitió observar si algún elemento se había situado fuera del rango de conveniencia, si se habían valorado todos los elementos de la misma forma con respecto a un determinado constructo y, lo que es muy importante, permitió establecer una comparación del elemento personal con el ideal.

Posteriormente se procedió a pasar los símbolos adoptados a valores numéricos, Io que permitió un análisis factorial con ordenador, utilizando el programa PM4 del paquete estadístico BMDP (Dixon 1981). Así obtuvimos las matrices de correlación, los constructos doblemente implicados y los factores principales.

Para el estudio de la evolución se construyeron rejillas de cambio, que fueron utilizadas en la entrevista final.

En el análisis de los diarios, desde la perspectiva del participante, suministrada por la técnica de rejillas, se identificaron unidades semánticas, que se sistematizaron en dimensiones, usando las obtenidas de las rejillas y recurrencias temáticas señaladas en la bibliografía por distintos autores (Zabalza 1986, Montero 1986), que se revelan como importantes cuando se analizan los diarios.

Por último, la transcripción de la entrevista final se organizó por categorías, sacadas de las rejillas y diarios, y variables (constructos), permitiendo una búsqueda de relaciones con las implicaciones obtenidas de los otros instrumentos de investigación, lo que permitió una verificación y enriquecimiento de la imagen obtenida mediante la técnica de rejillas.

\section{RESULTADOS}

Los resultados obtenidos muestran las imágenes de dos estudiantes para maestros cuyas concepciones personales y profesionales son muy distintas en las áreas de conocimiento consideradas. Estas diferencias les ha- 
figura 2

Importancia de la falta de conocimiento de la materia específica percibida por el alumno $A$.

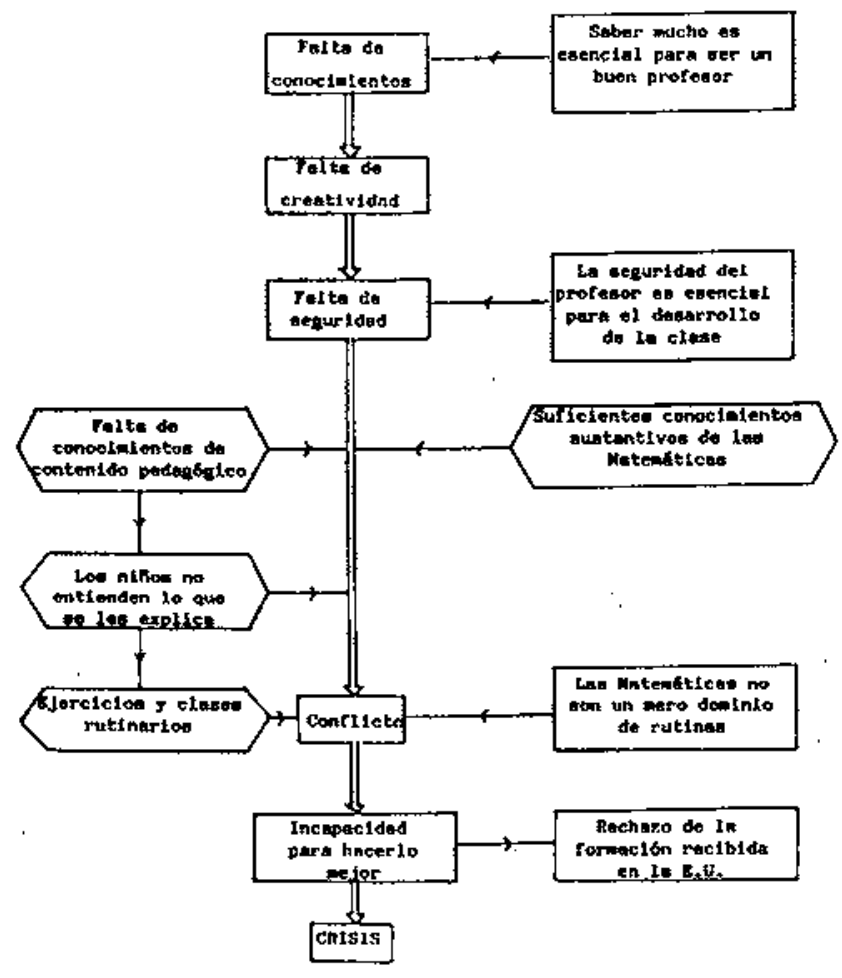

cen percibir de un modo completamente distinto su experiencia en las prácticas y, consecuentemente, dicha experiencia produce efectos may dispares en ellos.

Su interpretación personal de la realidad vivida durante las prácticas está muy influenciada por la percepción que tienen sobre sus conocimientos de Matemáticas (de la materia específica, de contenido pedagógico y curricular), que consideran muy limitados.

En particular, la percepción de la falta de conocimientos tiene efectos muy distintos en los dos participantes. Para el alumno A la amplitud y profundidad de conocimientos es algo indispensable para ser un buen profesor. Su visión de sí mismo como carente de ellas le lleva a asignarse también una falta de creatividad y, globalmente, una falta de seguridad. Y, para él, la seguridad del profesor es otro requisito indispensable para el desarrollo de la clase (figura 2).

Éste es el estado en el que acude al segundo período de prácticas. Su interpretación de las experiencias allí vividas no le tace percibir una insuficiencia de conocimientos sustantivos de las Matemáticas, pero sí de conocimiento de contenido pedagógico. Él asocia esta insuficiencia con el hecho de que los niños no son capaces de entender lo que él les explica, lo que le lleva a la realización de ejercicios rutinarios y al desarrollo de clases monótonas.

Esta realidad está en contradicción con su concepción de las Matemáticas como algo que debe ser asimilado figura 3

El papel del conocirniento en las prácticas del alumno $B$.

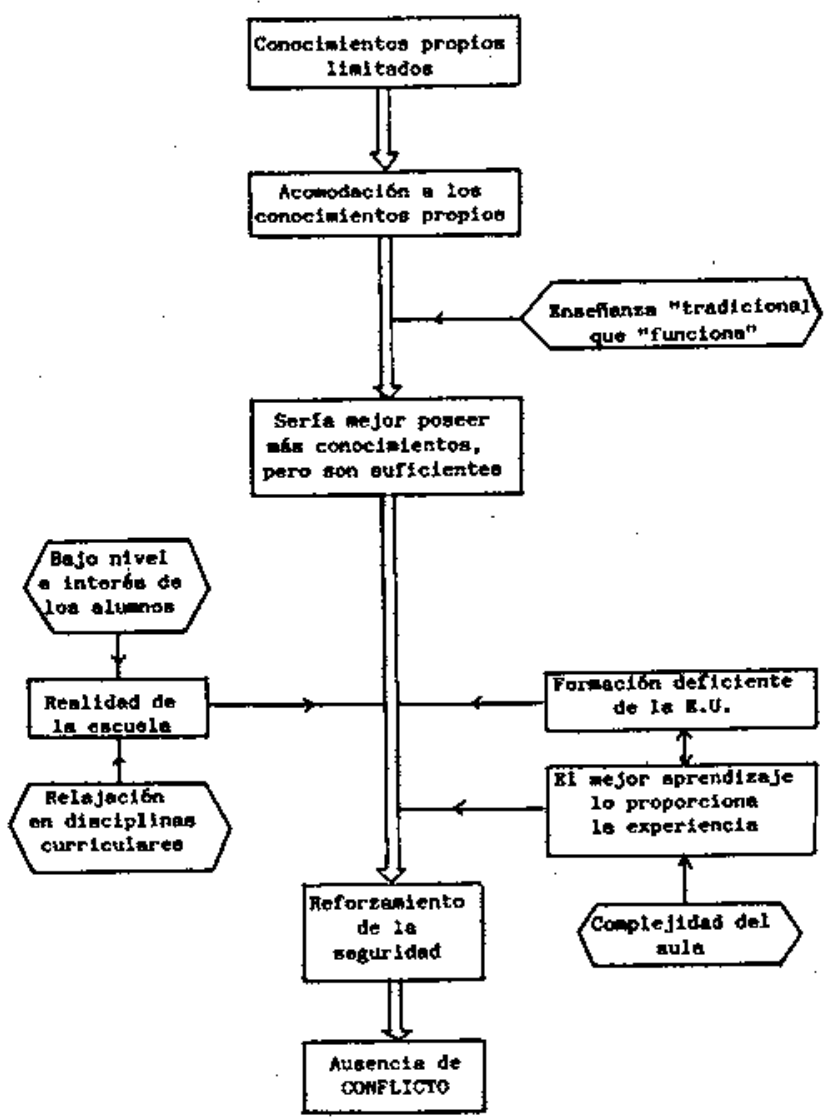

por los niños, y le origina un fuerte conflicto, ya que, además, se ve incapaz de hacerlo mejor en el futuro. Colateralmente, manifiesta un fuerte rechazo a la formación recibida en la Escuela Universitaria, ya que, bajo su perspectiva, no le ha suministrado los medios para desarrollar de un modo apropiado su tarea.

EI proceso es muy diferente para el alumno B (figura 3). Parte de la limitación de sus conocimientos y la necesidad de adecuar a ellos su práctica. Para ello recurre a su propia experiencia. La práctica le muestra que el tipo de enseñanza "tradicional" que han utilizado con él como alumno le funciona también como profesor. Su visión entonces es que sería mejor, desde luego, poseer más conocimientos, pero que puede desarrollar su labor correctamente sin ellos.

Además, una serie de factores externos le hacen sentirse no responsable de la situación. Por un lađo, está la realidad de la escuela en que desarrolla sus prácticas, con el bajo nivel e interés de los alumnos, y la insuficiente atención que percibe que se da a las Matemáticas.

Por otro lado, está la formación deficiente que piensa que ha recibido en la Escuela Universitaria. Además, la complejidad que percibe en el aula implica para él que 
la experiencia es el mejor camino, y casi el único, para adquirir los conocimientos que necesita. Esto tiene dos consecuencias. Le evita sentir una frustracion excesiva por lo que cree mala formación recibida, y le refuerza su seguridad como futuro maestro. Lo que no sabe, ya lo aprenderá. De este modo, evita eficazmente la aparición de un conflicto, como el que vimos en el caso anterior.

Aunque, por brevedad, los resultados que aquí hemos presentado son sólo una parte del trabajo total, sirven para mostrar las imágenes de dos estudiantes para maestros cuyas concepciones personales y profesionales son muy distintas en las áreas de conocimiento consideradas. Y no nos referimos sólo a las concepciones consideradas aisladamente, sino, y esto es lo más importante, al modo en que están insertas en los sistemas de constructos de cada uno de los participantes.

Antes de las prácticas de enseñanza, los dos participantes se anticipan a sí mismos como profesores en una forma altamente positiva, situándose muy próximos a su ideal. Hay que señalar que tienen una visión muy idealizada de la enseñanza. Sin embargo, la falta de conocimientos percibida, juega un papel primordial en las prácticas, aunque sus efectos son muy distintos en cada uno de ellos.

El alumno B considera que esta falta de conocimientos es una realidad que simplemente debe asumir, ya que el único medio para llegar a ser un buen profesor de Mate. máticas es la experiencia, percepción corroborada por los acontecimientos vividos durante sus prácticas. Con ello, no sólo evita la aparición de un conflicto, sino que refuerza su seguridad. De hecho, su sistema de constructos es estructurado y reestructurado de manera que evite la aparición de conflictos.

Desde un punto de vista estratégico, y utilizando los tếrminos de Lacey (1977), combina en gran medida voluntariamente el ajuste interiorizado y la sumisión estratégica.

El proceso seguido por el alumno A es totalmente distinto. Para él, la valídación de su actuación está en sí mismo. La limitación de sus conocimientos es una responsabilidad personal. Si no se considera capacitado para ser un buen maestro, ningún agente externo será capaz de transmitirle confianza.

Por otro lado, no está dispuesto a modificar su ideal de enseñanza. La consecuencia es un alejamiento de su anticipación como profesor del ideal, lo que le lleva a un conflicto. A diferencia del alumno B, no cree en la experiencia como un agente suficiente y casi único para la adquisición de conocimientos, Io que le lleva a mantener una postura mucho más crítica respecto de la formación recibida.

Centrándonos en las prácticas, mientras que para el alumno B resultan completamente irrelevantes, para el A representan la manifestación de un conflicto. Ámbos resultados son perfectamente visibles en los distintos instrumentos de investigación utilizados, y fueron además reconocidos explícitamente por cada uno de los participantes.

En este sentido, queremos insistir en la importancia de Ios diarios y entrevistas como medio para contrastar y validar los resultados obtenidos del análisis de las rejillas.

Para terminar, nuestro estudio pone de manifiesto la importancia que tiene el conocimiento, en sentido amplio, de las Matemáticas y su enseñanza en la forma de desarrollar su labor los maestros y los esudiantes para maestros. Más aún, hemos podido constatar el papel esencial que juega el conocimiento propio, tal y como es percibido por ellos, y la forma en que dicha percepción es interpretada e incorporada a su forma de estructurar la realidad.

Creemos que esta autopercepción de conocimientos suficientes es una condición indispensable para que un profesor se sienta, en alguna medida, identificado con un currículo y responsable de su desarrollo.

Con respecto a la asignatura de Matemáticas, la percepción que tienen los estudiantes para maestros de la relativa ineficacia de los cursos de Matemáticas Superiores de la Escuela Universitaria es algo que merece reflexión. El currículo de la E.U. tiende a aumentar los conocimientos de Matemáticas mediante la introducción de nuevos campos e ideas, y no desarrollando sus concepciones de las Matemáticas de Primaria y Secundaria (Owens 1987).

Estos nuevos conocimientos no son vistos como relevantes por los futuros maestros, y éste es el punto esencial. ¿Se rediseñan los programas de forma que se tengan en cuenta estas concepciones o se profundiza en la adquisición de nuevos conocimientos? Creemos que éste debe ser un motivo de reflexión.

Por último, queremos expresar nuestro agradecimiento a los participantes en este trabajo. Su vocación, estímulo e ilusión fueron un ejemplo para nosotros.

\section{RECONOCIMIENTOS}

Agradecemos a los profesores Luis Migue! Villar Angulo y Carlos Marcelo García las sugerencias y comentarios realizados durante el desarrollo de este trabajo. 


\section{REFERENCIAS BIBLIOGRÁFICAS}

BALL, D. L., 1989. Research on Teaching Mathematics: Making Subject Matter Knowledge Part of the Eqtation, en Brophy, J. (ed.), Advances in Research on Teaching, Vol. 2, Greenwich, (CT: JAI Press).

CALDERHEAD, J., 1987. Exploring Teachers' Thinking (Introduction). (Cassell Education: London).

CLARK, C. y PETERSON, P.L., 1986. Teachers' Thought Processes, en Wittrock, M.C. (ed.), Handbook of Research on Teaching. (Macmillan: New York).

COHEN, L. y MANION, L., 1980. Research Methods in Education. (Croom Helm: London).

COONEY, T.J., 1980. Research on teaching and teacher education, en Shumway, R. Y. (ed.), Research in Mathematics Education, Reston, VA, NCTM.

DIXON, W., I98 I. BMDP. Biomedical Computer Programs. Universidad de California.

ELBAZ, F., HOZ, Y., TOMER, R., CHAYOT, S., MAHLER, S. y YEHESKEL, N., 1986. The Use of Concepts Mapping in the Study of Teacher's Knowledge Structures, en Ben Peretz, Bromme y Halkes (eds.), Advances of Research on Teacher Thinking. (Sweets y Zeitlinger: Lisse).

ERICKSON, F., 1986. Qualitative Methods in Research on Teaching, en Wittrock, M.C. (ed.), Handbook of Research on Teaching. (Macmillan: New York).

FEIMAN-NEMSER, S. y FLODEN, R.E., 1986. The CuIture of Teaching, en Wittrock, M.C. (ed.), Handbook of Research on Teaching. (Macmillan: New York).

FRANSELLA, F. y BANNISTER, D., 1977. A manual for Repertory Grid Technique. (Academic Press: London).

GOODMAN, J., 1985. Field-based experience: A Study of social control and student teachers' response to institutional constrains, Journal of Education for Teaching, Vol. 11(1).

HARVEY, P.L., 1986. Student Teachers' Articulated Beliefs and their Congruence with Classroom Practices. Paper presented at the annual meeting of the AERA, San Francisco.

KELLY, G.A., 1955. The Psychology of Personal Constructs. (Norton: New York).

LACEY, C., 1977. The Socialization of Teachers. (London: Methuen).

LLINARES, S., 1989. Las creencias sobre la naturaleza de las Matemáticas y su enseñanza en estudiantes para profesores de Primaria: dos estudios de casos. Tesis doctoral inédita. Universidad de Sevilla.

LLINARES, S. y SANCHEZ, M.V., 1986. Las creencias sobre las Matemáticas y la enseñanza de las Matemáticas en profesores de EGB en formacion, en Villar Angulo (ed.), Pensamiento de los profesores y Toma de decisiones. (Servicio de Publicaciones, Universidad de Sevilla).

LLINARES, S. y SÁNCHEZ, M.V., 1989. La representación del conocimiento del profesor: una agenda de investigacion. Comunicación presentada a las $\mathrm{V}$ Jornadas Andaluzas de Profesores de Matemáticas de Escuelas de Magisterio. Granada.
LLINARES, S. y SÁNCHEZ, M.V., (en prensa). El conocimiento profesional del profesor y la enseñanza de las Matemáticas, en Llinares y Sánchez (eds.), Lecturas sobre la relación Teoría-Práctica en Educación Matemática. (Sevilla: Alfar).

LLINARES, S. y SÁNCHEZ, M.V. (en prensa). Las creencias epistemologicas sobre la naturaleza de las Matemáticas y su enseñanza y el proceso de llegar a ser un profesor, Revista de Educación.

MARCELO, C., 1987. El pensamiento del profesor. (Barcelona: CEAC),

McQUALTER, J.W., 1986. Becoming a Mathematics Teacher: The use of Personal Construct Theory, Educational Studies in Mathematics, 17(1), pp. 1-14.

MONTERO, M.L., 1986. Pensamiento de los profesores. Investigación cualitativa y formación del profesorado, en Villar Angulo (ed.), Pensamiento de los profesores y Toma de decisiones. (Servicio de Publicaciones. Universidad de Sevilla).

MORINE-DERSHIMER, G., 1983. Tapping teacher thinking trough triangulation of data sets. Report Published by Research and Development Center for Teacher Education.

OWENS, J.E., 1987. A Study of four Preservice Secondary Mathematics Teachers' Constructs of Mathematics and Mathematics Teaching. DoctoraI Dissertation. University of Georgia.

POPE, M.L. y KEEN, T.R., 1981. Personal Construct Psychology and Education. (London: Academic Press).

SÁNCHEZ, M.V., 1989. Los constructos de dos estudiantes para profesores de primaria acerca de las Matemáticas y su enseñanza. Influencia de las prácticas. Tesis doctoral inédita. Universidad de Sevilla.

SÁNCHEZ, M.V., y LLINARES, S., 1987. Un estudio de la influencia de los períodos de prácticas sobre el pensamiento de los futuros maestros. Actas de las V Jornadas de estudio sobre la investigación en la Escuela, Sevilla.

SANCHEZ, M.V. y LLINARES, S., 1988. Un estudio de las creencias del futuro maestro en relación a Ias Matemáticas. Influencia de las prácticas, en Marcelo, C. (ed.), Avances en el estudio del pensamiento de los profesores. (Publicaciones de la Universidad de Sevilla).

SHULMAN, L.S., 1986a. Paradigms and Research Programs in the Study of Teaching: A Contemporany Perspective, en Wittrock, M. C. (ed.), Handbook of Research on Teaching. (New York: Macmillan).

SHULMAN, L.S., 1986b. Those who Understand: Knowledge Growth in Teaching, Educational Research, Fe: bruary, pp. 4-14.

SHULMAN, L.S., 1987a. Sounding an Alarm: A reply to Sockett, Harvard Educational Review, Vol. 57(4), pp. $473-482$.

SHULMAN, L.S., 1987b. Knowledge and Teaching: Foundations of the New Reform, Harvard Educational Review, Vol. 57(1), pp. 1-22.

THOMPSON, A.G., 1984. The Relationship of Teacher's conceptions of Mathematics and Mathematics teaching 
for instrucional practice, Educational Studies in Mathematics, 15, pp. 105-127.

VAN HUNEN, W., 1986. Describing teacher cognitions with the repgrid: some methodological problems. Proceedings of the Third Conference on Teacher Thinking and Proffesional Action, Leuven University, Beigium.

VEENMAN, S., 1984. Perceived Problems of Beginning Teachers, Review Research, 54(2), pp. 143-178.

WILSON, S.M., SHULMAN, L.S. y RICHERT, A.E., 1987. 150 Differents Ways of Knowing: Representations of
Knowledge in Teaching, en Calderhead (ed.), Exploring Teachers' Thinking. (London: Cassell Education).

ZABALZA, M.A., 1986. El paradigma del pensamiento del profesor y sus aplicaciones a la formación y desarrollo profesional del profesorado: trabajo cualitativo con dia. rios de profesores. Comunicación presentada al I International Meeting on Psychological Teacher Education. Braga, Portugal.

ZEICHER, K. y GORE, J., 1989. Teacher socialization, en Houston, W.R., Haberman, M. y SikuIa, J. (eds.), Handbook of Research on Teacher Education. (New York: Macmillan). 